Check for updates

Cite this: Chem. Commun., 2017, 53,4830

Received 22nd December 2016, Accepted 29th March 2017

DOI: $10.1039 / \mathrm{c} 6 \mathrm{cc} 10179 \mathrm{~h}$

rsc.li/chemcomm

\section{Unprecedented thermal condensation of tetracyanocyclopropanes to triazaphenalenes: a facile route for the design of novel materials for electronic applications $\dagger$}

\author{
Iliya E. Kuznetsov, ${ }^{a}$ Diana K. Susarova, ${ }^{a}$ Lyubov A. Frolova, ${ }^{a}$ \\ Alexander S. Peregudov, ${ }^{b}$ Alexander F. Shestakov, ${ }^{a}$ Sergey I. Troyanov, ${ }^{c}$ \\ Keith J. Stevenson ${ }^{d}$ and Pavel A. Troshin (D) $*$ da
}

\begin{abstract}
We report an unusual thermal condensation of readily available tetracyanocyclopropanes to tetracyanosubstituted triazaphenalenes, which revealed interesting optoelectronic properties such as strongly pronounced solvatochromism and bright photoluminescence. Optical memory elements and organic light emitting diodes with a deep red electroluminescence were designed using triazaphenalenes, thus highlighting the potential of these compounds as materials for electronic applications.
\end{abstract}

Macrocyclic and condensed polycyclic aromatic compounds bearing multiple electron withdrawing substituents have attracted much attention over several decades. ${ }^{1}$ Perfluorinated phthalocyanines and fluorine-loaded pentacene derivatives were applied successfully as air-stable organic semiconductors enabling reliable long-term operation of organic field-effect transistors (OFETs) under ambient environment. ${ }^{2}$ Organic semiconductors based on perylene and naphthalene diimides, indigo and isoindigo molecules and other fused polycyclic compounds bearing strongly electron withdrawing cyano groups have been intensively explored as promising materials for organic electronics. ${ }^{3}$ Phthalocyanines bearing eight cyano groups demonstrated facile electrochemical reduction to the corresponding anions, which were utilized in the design of novel materials with exciting optical, electrical and magnetic properties. ${ }^{4}$ Finally, macrocyclic compounds loaded with multiple $\mathrm{CN}$ groups demonstrated good electrochemical performance while being used as cathode materials in lithium batteries. ${ }^{5}$

${ }^{a}$ IPCP RAS, Semenov Prospect 1, Chernogolovka, 142432, Russia.

E-mail: troshin2003@inbox.ru; Fax: +7 496522-3507; Tel: +7 4965221418

${ }^{b}$ INEOS RAS, Vavylova St. 28, B-334, Moscow, 119991, Russia

${ }^{c}$ Department of Chemistry, Moscow State University, Leninskie gory, Moscow, 119991, Russia

${ }^{d}$ Skolkovo Institute of Science and Technology, Nobel St. 3, Moscow, 143026, Russian Federation

$\dagger$ Electronic supplementary information (ESI) available: NMR and ESI MS spectra for all compounds, crystallographic data for 1, UV-vis and PL spectra for selected compounds. CCDC 1522029. For ESI and crystallographic data in CIF or other electronic format see DOI: $10.1039 / \mathrm{c} 6 \mathrm{cc} 10179 \mathrm{~h}$
Triazaphenalene and its functional derivatives possessing a highly attractive conjugated electronic $\pi$-system incorporating a central nitrogen lone electron pair also can be considered as promising materials for organic electronics and energy storage devices. Unfortunately, the exploration of this family of compounds was limited severely by the complexity of their synthesis. The previously reported procedures are based on using complex precursor molecules and require several steps for preparing the target products. ${ }^{6}$ Moreover, the existing methods can hardly be used for preparing triazaphenalene derivatives bearing multiple electron deficient substituents, e.g. several cyano groups.

In this communication we present a serendipitously discovered thermal condensation reaction of tetracyanocyclopropanes yielding 1,4,9b-triazaphenalenes bearing four $\mathrm{CN}$, two aryl and single amine substituents (Scheme 1).

The reaction proceeds with heating the solutions of 3-arylcyclopropane-1,1,2,2-tetracarbonitriles at reflux in 1,2-dichlorobenzene for $4-20 \mathrm{~h}$. The colorless precursor solutions turn to deep red or reddish-brown and dark precipitates are formed while the reaction is progressing. The crude reaction mixtures were extracted with a sufficient volume of warm toluene and processed by column chromatography on silica to isolate the

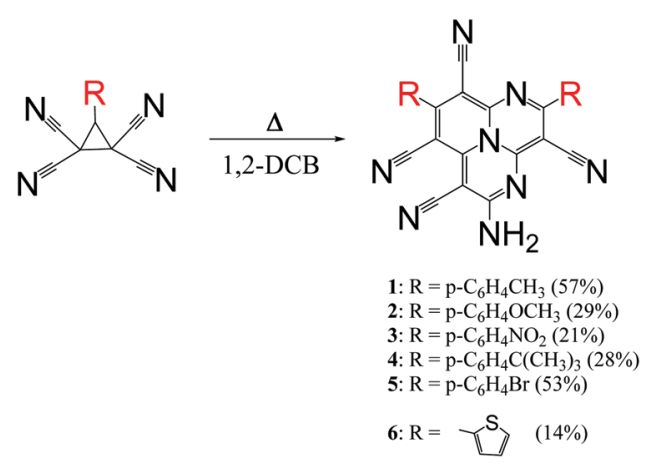

Scheme 1 Synthesis of the substituted 1,4,9b-triazaphenalenes. 
target 5-amino-2,8-bisaryl-1,4,9b-triazaphenalene-3,6,7,9-tetracarbonitriles 1-6 (referred to as triazaphenalenes below; see details in the ESI $\dagger$ ). The yields of the triazaphenalenes are limited mostly by the formation of some more complex condensation by-products identified by ESI MS (Fig. S1, ESI $\dagger$ ). These products of formal trimerization and tetramerization of the corresponding tetracyanocyclopropanes were almost insoluble in common organic solvents, which prevented their purification and spectroscopic characterization. We emphasize that the modest isolated yields of the triazaphenalenes can still be considered acceptable since the starting 3-arylcyclopropane1,1,2,2-tetracarbonitriles are cheap and readily available substances. Indeed, they can be obtained in high yields from the corresponding aldehydes and malononitrile. ${ }^{7}$

It is rather clear that the thermal condensation of tetracyanocyclopropanes represents a complicated multistep process and revealing the exact mechanism of this reaction might be very challenging. Nevertheless, we proposed a plausible sequence of steps, which explains the formation of triazaphenalenes as the main reaction products (Scheme 2 ).

This mechanism is based on the assumption that tetracyanocyclopropane undergoes ring opening with the formation of the 1,3-dipolar intermediate I, undergoing a [2+3]cycloaddition reaction with other molecules of the starting cyclopropane, thus producing the bicyclic intermediate II. The ring opening in II affords the intermediate III, which undergoes intramolecular nucleophilic addition and ring closure, thus producing IV. The opening of the cyclopropane ring in IV accompanied by the release of a proton generates the active anionic intermediate $\mathbf{V}$, which undergoes nucleophilic addition and rearrangement yielding VI. Finally, VI undergoes additional intramolecular nucleophilic addition producing VII, which accepts a proton and isomerizes into the more thermodynamically stable 5-amino-2,8-bisaryl-1,4,9btriazaphenalene-3,6,7,9-tetracarbonitrile. We also observed that

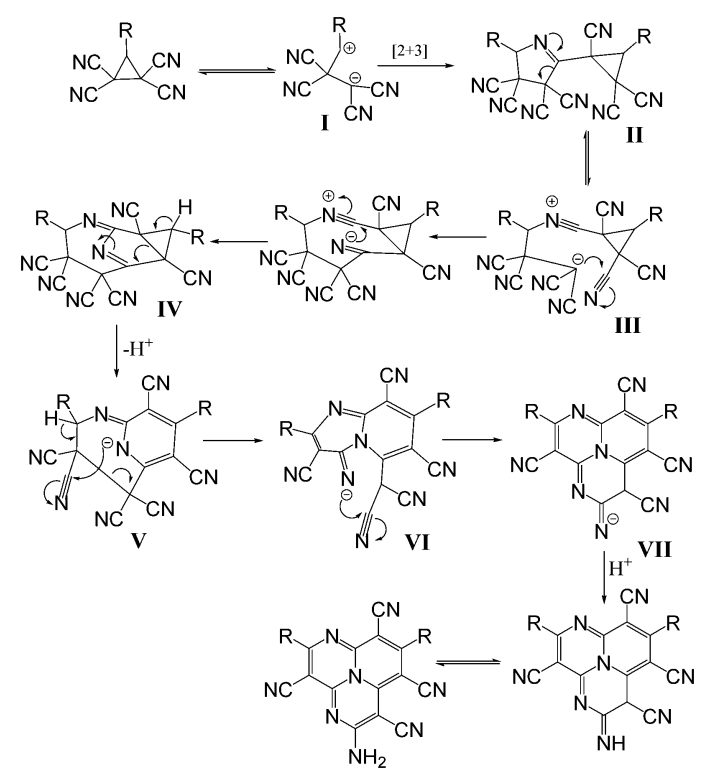

Scheme 2 Proposed mechanism of the reaction.

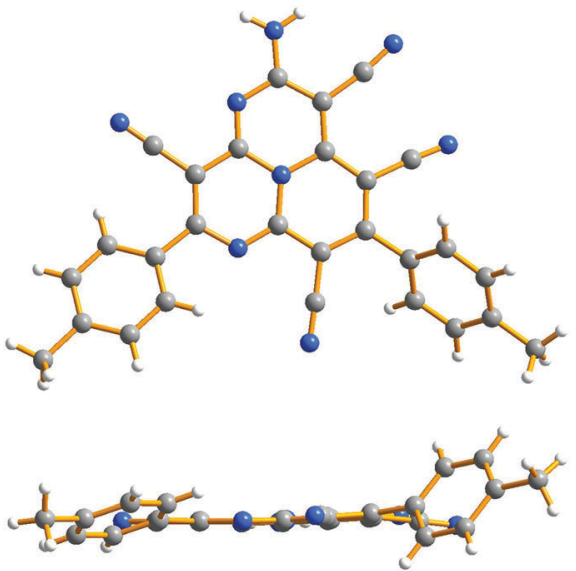

Fig. 1 Two projections of the molecule of 1 according to the X-ray single crystal diffraction data.

the introduction of strong acids such as $p$-toluenesulfonic acid inhibits the reaction presumably due to the protonation of active anion intermediates. The introduction of organic bases (e.g. DBU) accelerates the reaction, but strongly decreases its selectivity.

Revealing the molecular structures of triazaphenalenes was strongly facilitated by successful X-ray single crystal structure determination for compound 1 (Fig. 1). The triazaphenalene core was found to be almost planar thus featuring an unusual geometry of the central ternary amine fragment. All cyano and amine substituents are coplanar with the core tricyclic system, while the aromatic rings are twisted with the dihedral angles defined by the planes of the triazaphenalene and phenyl rings equal to 20 and 53 degrees.

All isolated triazaphenalenes 1-6 were extensively characterized using electrospray mass spectra (ESI MS), ${ }^{1} \mathrm{H}$ and ${ }^{13} \mathrm{C}$ NMR spectra, as well as H-H COSY, H-C HSQC and H-C HMBC 2D correlations (Fig. S2-S16, ESI $\dagger$ ), which confirmed unambiguously the molecular composition and structures of compounds 1-6.

It was particularly interesting to observe the solvation of triazaphenalenes by NMR spectroscopy (Fig. S17, ESI $\dagger$ ). The ${ }^{1} \mathrm{H}$ NMR spectrum of compound 1 recorded in $\mathrm{CDCl}_{3}$ shows a broad signal at $\sim 6.3 \mathrm{ppm}$, corresponding to the $\mathrm{NH}_{2}$ protons. However, the NMR spectrum recorded from the DMSO-D6 solution of the same compound exhibits two distinct and strongly low-field shifted signals $(1 \mathrm{H})$ for non-equal $\mathrm{N}-\mathrm{H}$ protons (Fig. S17, ESI $\dagger$ ).

The $2 \mathrm{D}{ }^{1} \mathrm{H}^{-15} \mathrm{~N}$ HMBC spectrum of compound 1 in DMSO revealed that the signals of the $\mathrm{N}-\mathrm{H}$ protons at 8.61 and $9.23 \mathrm{ppm}$ are both coupled to the same ${ }^{15} \mathrm{~N}$ signal (Fig. S18, ESI $\dagger$ ). This evidences that compound $\mathbf{1}$ does not undergo any tautomerization in polar solvents and the observed spectral changes are related to its strong solvation with DMSO molecules.

Quantum chemical DFT calculations (see details in the ESI $\dagger$ ) allowed us to reproduce the molecular geometry of compound 1 with high precision (Fig. S19, ESI $\dagger$ ). The mean absolute deviation of the calculated bond lengths from the experimental values was around $0.01 \AA$. The dihedral angles defined by the planes of the triazaphenalene system and phenyl rings were 
24 and 51 degrees, thus corresponding well to the experimental values given above.

Importantly, the DFT calculations have revealed a high energy barrier of $80.0 \mathrm{~kJ} \mathrm{~mol}^{-1}$ for the amine group rotation (Fig. S20, ESI $\dagger$ ). This effect is caused by the shortening of the $\mathrm{C}-\mathrm{NH}_{2}$ bond in $\mathbf{1}$ due to the conjugation of the nitrogen lone electron pair with the strongly electron-deficient triazaphenalene ring. The restricted rotation of the amine group explains the non-equality of the two $\mathrm{NH}_{2}$ protons revealed in the ${ }^{1} \mathrm{H}$ NMR spectra.

Theoretical calculations have shown that DMSO forms strong hydrogen bonds with the $\mathrm{H}$ atoms of the $\mathrm{NH}_{2}$ group ( $\mathrm{H} \cdots \mathrm{O}$ distances are in the range of 1.55-2.11 $\mathrm{A}$ ). Solvation results in elongation of the $\mathrm{N}-\mathrm{H}$ bonds by $0.005-0.068 \AA$ and leads to an increase of the energy barrier for amine group rotation by $8.1 \mathrm{~kJ} \mathrm{~mol}^{-1}$. The calculations also revealed that the solvation of 1 with DMSO induces a significant (by 3-4 ppm) low-field shift of the NMR signals corresponding to the $\mathrm{NH}_{2}$ protons, which was observed experimentally.

The strong intermolecular interactions of triazaphenalenes with solvent molecules resulted in interesting solvatochromic effects in their UV-vis absorption and photoluminescence (PL) spectra. Fig. 2 shows the spectra of compound 2 recorded in different solvents (concentration of $\mathbf{2}$ was identical for all solutions).

The change of the solvent induces notable shifts in the absorption and emission bands, molar extinction and PL intensity. This kind of behavior might find practical applications, e.g., in the development of fluorescent probes for certain molecular or ionic species. ${ }^{8}$ It should be also mentioned that the optoelectronic properties of these compounds can be tuned by appropriate modification at the aryl groups, as can be concluded from the comparison of the UV-vis and PL spectra of differently substituted triazaphenalenes (Fig. S21, ESI $\dagger$ ). No significant changes in the absorption spectra occur while going from solution to the solid films (spectrum of $\mathbf{1}$ is given as an example in Fig. S22, ESI $\dagger$ ).

The observed "switching" behavior of triazaphenalenes in solution can be also realized in the solid films, which opens wide opportunities for designing different types of data storage devices. We have succeeded in the application of triazaphenalene $\mathbf{5}$ as a light-sensitive material for the construction of photoswitchable organic field-effect transistors (OFETs), operating as optical memory elements. The architecture of these devices is shown schematically in Fig. 3. Briefly, an aluminum oxide dielectric was grown electrochemically on aluminum gate electrodes.
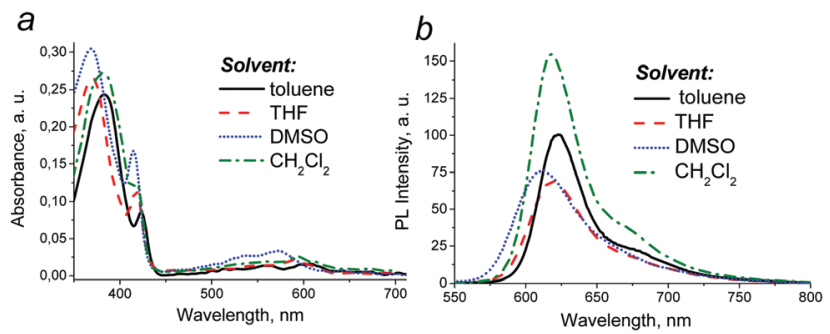

Fig. 2 UV-vis (a) and PL (b) spectra of $\mathbf{2}$ in different solvent media.

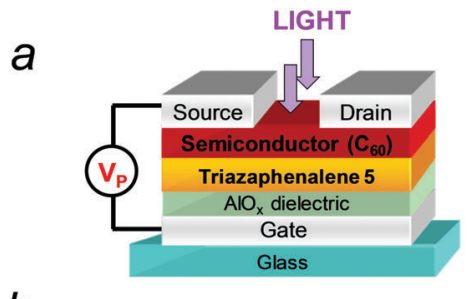

$b$
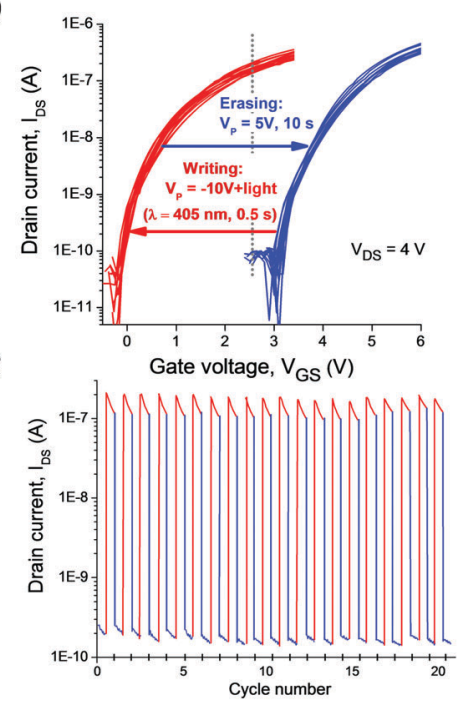

Fig. 3 Schematic layout of the memory element incorporating compound 5 (a), transfer characteristics of the device while being switched between two distinct electrical states (b), and write-read-erase cycling behavior (c).

Triazaphenalene 5 was spin-coated from the DMF solution $\left(10 \mathrm{mg} \mathrm{ml} \mathrm{m}^{-1}, 1000 \mathrm{rpm}\right)$ on the top of the $\mathrm{AlO}_{x}$ dielectric. A semiconductor layer $(100 \mathrm{~nm})$ was grown by the thermal evaporation of [60]fullerene under vacuum. The devices were completed by the deposition of the $100 \mathrm{~nm}$ thick silver source and drain electrodes through a shadow mask (details are given in the ESI $\dagger$ ).

Programming of the devices was accomplished by applying a positive bias voltage between the source and the gate electrodes $\left(V_{\mathrm{GS}}=5 \mathrm{~V}\right.$, defined as $V_{\mathrm{P}}$ in Fig. 3a) for $10 \mathrm{~s}$ (writing regime) or simultaneous application of the negative bias voltage $\left(V_{\mathrm{GS}}=-10 \mathrm{~V}\right)$ and a light pulse $(\lambda=405 \mathrm{~nm}$ ) for $0.5 \mathrm{~s}$ (erasing regime). Previously we have developed similar operation regimes for optical memory elements based on organic photochromic molecules. ${ }^{9}$ The OFETs incorporating the light- and field-sensitive triazaphenalene 5 can be reversibly switched many times between two distinct electrical states, as it is shown in Fig. 3b. It should be emphasized that these electrical states are characterized by very different $I_{\mathrm{DS}}$ currents at $V_{\mathrm{GS}}=2.55 \mathrm{~V}$, thus delivering an impressive switching coefficient $K_{\mathrm{SW}}=I_{\mathrm{DS}}(1) / I_{\mathrm{DS}}(2)>2000$. Additionally, the fabricated devices demonstrated a good write-read-erase cycling behavior (Fig. 3c), characteristic of advanced organic memories. The observed remarkably high photo/electrical switching effect is apparently related to the intrinsic properties of triazaphenalenes (e.g. light- and field-induced electron transfer, perhaps involving also a semiconductor material, with the subsequent charge trapping; see examples in ref. 9) and deserve more thorough exploration in the future. 
a

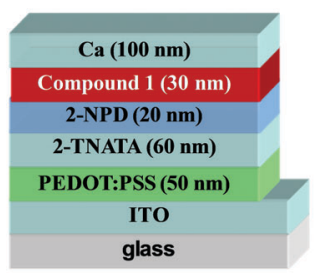

$b$

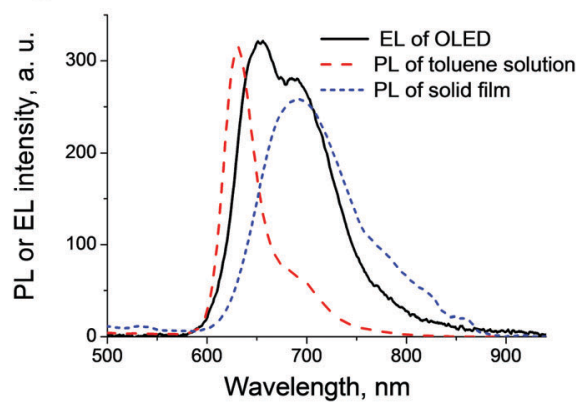

Fig. 4 Schematic layout of the OLED architecture (a) and comparison between the PL spectrum of compound $\mathbf{1}$ in solution and the OLED EL spectrum (b).

The intense photoluminescence of triazaphenalenes in solution and in the solid state allows one to consider them as promising materials for organic light-emitting diodes (OLEDs). We have fabricated a simple OLED architecture, as shown in Fig. 4a. A thin film of the hole-injection material PEDOT:PSS was spin-coated on the top of an indium-tin oxide (ITO) covered glass. Afterwards, the hole transport layers of $4,4^{\prime}, 4^{\prime \prime}$-tris[2-naphthyl(phenyl)amino]triphenylamine (2-TNATA) and $N, N^{\prime}$ di(1-naphthyl)- $N, N^{\prime}$-diphenyl-(1,1'-biphenyl)-4, $4^{\prime}$-diamine (NPD) were deposited by vacuum evaporation. Triazaphenalene 1 used as an electroluminescent material was evaporated on the top of 2-TNATA and the device structure was completed by depositing a low work function calcium cathode.

The fabricated OLEDs showed turn-on voltages of $\sim 3.5 \mathrm{~V}$ and demonstrated a maximal brightness of $\sim 1000 \mathrm{~cd} \mathrm{~m}^{-2}$ at $\sim 8.0 \mathrm{~V}$. The current efficiency of the OLEDs was below $0.5 \mathrm{~cd} \mathrm{~A}^{-1}$, which might be related to significant non-radiative recombination losses in the non-optimized device architecture. The electroluminescence (EL) spectrum of the OLEDs was found to be significantly broadened and red-shifted as compared to the PL spectrum of the toluene solution of 1 (Fig. 4b). This effect might be related to the formation of exciplexes or electroplexes appearing due to aggregation and strong intermolecular interactions of triazaphenalene molecules in the solid state.

In conclusion, we have developed a facile synthetic route for the preparation of 5-amino-2,8-bisaryl-1,4,9b-triazaphenalene3,6,7,9-tetracarbonitriles, which can hardly be obtained using other methods. These compounds exhibited a unique combination of physical, optical and electronic properties, pointing towards numerous potential applications. We have demonstrated optical memory elements and deep-red OLEDs based on some of the synthesized triazaphenalenes. Considering the molecular structure of triazaphenalenes, one can also envisage their successful utilization as organic cathode materials for lithium and sodium batteries with high theoretical capacities (e.g. $\sim 350 \mathrm{~mA} \mathrm{~h} \mathrm{~g}{ }^{-1}$ for compound 1). This aspect is currently under investigation in our laboratories.

This work was supported by Russian Science Foundation (grant no. 16-13-00111). We acknowledge Dr A. N. Vereshchagin and Dr M. N. Elinson of N. D. Zelinsky Institute of Organic Chemistry of RAS for providing some of the starting cyclopropanes. PAT gratefully thanks Prof. S. M. Igumnov of INEOS RAS for valuable discussions.

\section{Notes and references}

1 J.-H. Dou, Y.-Q. Zheng, Z.-F. Yao, T. Lei, X. Shen, X.-Y. Luo, Z.-A. Yu, S.-D. Zhang, G. Han, Z. Wang, Y. Yi, J.-Y. Wang and J. Pei, Adv. Mater., 2015, 27, 8051-8055; H.-J. Yun, S.-J. Kang, Y. Xu, S. O. Kim, Y.-H. Kim, Y.-Y. Noh and S.-K. Kwon, Adv. Mater., 2014, 26, 7300-7307; J. Xie, K. Shi, K. Cai, D. Zhang, J.-Y. Wang, J. Pei and D. Zhao, Chem. Sci., 2016, 7, 499-504.

2 J. M. Wasikiewicz, L. Abu-Sen, A. B. Horn, J. M. Koelewijn, A. V. S. Parry, J. J. Morrison and S. G. Yeates, J. Mater. Chem. C, 2016, 4, 7309-7315; H. Jiang, J. Ye, P. Hu, F. Wei, K. Du, N. Wang, T. Ba, S. Feng and C. Kloc, Sci. Rep., 2014, 4, 7573; M. C. R. Delgado, K. R. Pigg, D. A. da Silva Filho, N. E. Gruhn, Y. Sakamoto, T. Suzuki, R. M. Osuna, J. Casado, V. Hernández, J. T. L. Navarrete, N. G. Martinelli, J. Cornil, R. S. Sánchez-Carrera, V. Coropceanu and J.-L. Brédas, J. Am. Chem. Soc., 2009, 131, 1502-1512.

3 X. Zhang, Z. Wang, S. Chen, Z. Zhao, W. Yuan, H. Wang and X. Gao, Chin. J. Chem., 2014, 32, 1057-1064; J. Gao, C. Xiao, W. Jiang and Z. Wang, Org. Lett., 2014, 16, 394-397; C. Zhang, K. Shi, K. Cai, J. Xie, T. Lei, Q. Yan, J.-Y. Wang, J. Pei and D. Zhao, Chem. Commun., 2015, 51, 7144-7147; Q. Wu, X. Qiao, Q. Huang, J. Li, Y. Xiong, X. Gao and H. Li, RSC Adv., 2014, 4, 16939.

4 S. Sergeyev, E. Pouzet, O. Debever, J. Levin, J. Gierschner, J. Cornil, R. Gómez Aspe and Y. H. Geerts, J. Mater. Chem., 2007, 17, 1777-1784; T. G. Gopakumar, H. Tang, W. R. Thiel and M. Hietschold, J. Phys. Chem. C, 2008, 112, 7698-7705; H.-Q. Xiang, K. Tanaka, A. Takahara and T. Kajiyama, Langmuir, 2002, 18, 2223-2228.

5 Y. Asai, S. Miyata, K. Onishi, T. Arai, M. Matsumoto and K. Shigehara, Electrochim. Acta, 2000, 46, 77-81; Y. Hanyu, T. Sugimoto, Y. Ganbe, A. Masuda and I. Honma, J. Electrochem. Soc., 2013, 161, A6-A9; T. Matsunaga, T. Kubota, T. Sugimoto and M. Satoh, Chem. Lett., 2011, 40, 750-752.

6 Y. Matsuda, H. Gotou, K. Katou and H. Matsumoto, Chem. Pharm. Bull., 1989, 37, 1188-1191; K. Kurata, H. Awaya, H. Gotou, Y. Tominaga, Y. Matsuda and G. Kobayashi, Chem. Pharm. Bull., 1985, 33, 3034-3037; M. Kuya, K. Kurata, H. Awaya, Y. Tominaga, Y. Matsuda and G. Kobayashi, Chem. Pharm. Bull., 1978, 26, 680-681; O. Ceder and K. Vernmark, Acta Chem. Scand., Ser. B, 1977, 31, 235-238.

7 N. Noroozi Pesyan and M. Rezaee, Monatsh. Chem., 2014, 145, 1165-1171; A. N. Vereshchagin, M. N. Elinson, N. O. Stepanov and G. I. Nikishin, ISRN Org. Chem., 2011, 2011, 469453.

8 S. W. Thomas, G. D. Joly and T. M. Swager, Chem. Rev., 2007, 107, 1339-1386; O. S. Wolfbeis, J. Mater. Chem., 2005, 15, 2657.

9 L. A. Frolova, A. A. Rezvanova, V. Z. Shirinian, A. G. Lvov, A. V. Kulikov, M. M. Krayushkin and P. A. Troshin, Adv. Electron. Mater., 2016, 2, 1500219; L. A. Frolova, P. A. Troshin, D. K. Susarova, A. V. Kulikov, N. A. Sanina and S. M. Aldoshin, Chem. Commun., 2015, 51, 6130; L. A. Frolova, A. A. Rezvanova, B. S. Lukyanov, N. A. Sanina, P. A. Troshin and S. M. Aldoshin, J. Mater. Chem. C, 2015, 3, 11675-11680. 